\title{
Caracterização Biométrica dos Caprinos da República de Cabo Verde
}

Pires, L.C. ${ }^{\circledR}$; Machado, T.M. ${ }^{2}$; Fonseca, J. de D. ${ }^{3}$; Fonseca, J.F. ${ }^{4}$; Pile, E. ${ }^{5}$ e Brandão, F.Z. ${ }^{6}$

Universidade Federal do Sul da Bahia. Brasil.

2Universidade Federal de Viçosa. Minas Gerais. Brasil.

${ }^{3}$ Ministério do Ambiente, do Desenvolvimento Rural e dos Recursos Marinhos da República de Cabo Verde. Brasil.

${ }^{4}$ Empresa Brasileira de Pesquisa Agropecuária. Brasil.

5 Universidade do Panama. Brasil.

৫Universidade Federal Fluminense. Brasil.

\section{PaLAVRAS ChAVE ADICIONAIS}

Aptidão zootécnica.

Biometria.

Conservação animal.

Recursos genéticos.

\section{RESUMO}

Objetivou-se discernir populações caprinas de cinco ilhas da República de Cabo Verde (n=533) por meio de dados biométricos e análises estatísticas. Foram avaliadas 16 características de fêmeas adultas, através da estatística descritiva simples, análise de variância, teste de multicolinearidade, distância generalizada de Mahalanobis (D2) e algoritmo UPGMA (Unweighted Pair Group Method Arithmetic Mean). Após o teste de multicolinearidade foi identificada e descartada a variável profundidade torácica. As D2 foram calculadas com base nas 15 medidas biométricas. $O$ maior valor da $D^{2}$ foi entre as populações das ilhas do Fogo e São Nicolau (22,73), e a menor D2 foi entre Santo Antão e São Vicente $(3,71)$. O dendrograma a partir de 15 variáveis em cinco populações colocou as cabras da ilha de Fogo em ramo a parte das demais. Agruparam-se num ramo as cabras das ilhas de Santo Antão e São Vicente. Este resultado está de acordo com a distância geográfica entre as ilhas de Cabo Verde e o histórico recente de intercâmbio de animais entre elas.

\section{Biometric characterization of the goats in the Republic of Cape Verde}

\section{SUMMARY}

\section{ADDITIONAL KEYWORDS}

Zootechnical aptitude.

Biometry.

Animal conservation.

Genetic resources.

\section{INFORMATION}

\section{Cronología del artículo.}

Recibido/Received: 16.04 .2018

Aceptado/Accepted: 03.10.2019

On-line: 15.07.2019

Correspondencia a los autores/Contact e-mail:

luanna.ufsb@gmail.com
The aims in this study was test the genetic discernment among five goats populations of the Republic of Cape Verde ( $\mathrm{n}=533$ ) by biometric data and statistical analysis. It were analysed 16 characteristics in adult goats, through descriptive statistics, analysis of variance, test for multicollinearity, Mahalanobis distance (D2) and UPGMA algorithm (Unweighted Pair Group Method Arithmetic Mean). After the test of multicollinearity was identified and discarded the variable thoracic depth. The $D^{2}$ were calculated using 15 biometric measurements. The maximum value of $\mathrm{D}^{2}$ was between the populations of the islands of Fire and São Nicolau (22.73), and D² was lower between Santo Antão and São Vicente (3.71). The dendrogram based on biometric data in five populations put the goats on the Fire Island in other branch. Clustered in one the branch the goats from the islands of Santo Antão and São Vicente. This result is consistent with the geographic distance among the islands of Cape Verde and the recent history of exchange of animals among them.

\section{INTRODUÇÃO}

A existência de caprinos nas Ilhas de Cabo Verde tem sido conhecida desde o século 15, quando as ilhas foram colonizadas pelos portugueses. Os caprinos cabo-verdianos foram oriundos, no período colonial, de Portugal (Machado 2013, pp.11-8). É possível que a cabra saheliana tenha entrado na constituição da cabra destas ilhas (Pires et al. 2012, p.777). Segundo a documentação da época da colonização, as ilhas achavam-se despovoadas e sem vestígios de presença humana na altura da chegada dos portugueses, pelo que foi necessário trazer gente, gado e plantas para dar início a um processo de permanência dos habitantes iniciada em 
1462 (Carvalho \& Sá 2007, pp.124-5). Após a conquista, as ilhas tornaram-se uma importante estação de negociação marítima através da qual as raças caprinas foram introduzidas e, posteriormente, influenciaram as populações caprinas locais. O grau de influência varia de uma ilha para outra. Com o passar dos séculos, os caprinos cabo-verdianos, em cada ilha, evoluíram, em grande parte, de forma isolada. Estes animais não são reconhecidos oficialmente como uma raça.

Cabo Verde possui aproximadamente 215,9 mil cabeças caprinas (FAOSTAT, 2017). As ilhas de Santiago $(40,43 \%)$, Fogo $(17,74 \%)$ e Santo Antão (16,05\%) possuem maior efetivo caprino (República de Cabo Verde/ MADRRM, 2004). Embora a criação de caprinos seja algo comum nas famílias rurais cabo-verdianas, especialmente nas ilhas do Fogo, Santo Antão, São Nicolau, Santiago e São Vicente, ela é feita ainda em pequena escala e de forma rudimentar (Pecuária, 2011).

As características biométricas, como peso, altura, comprimento, perímetro escrotal, que precisam ser mensuradas com uso de instrumentos de medição, permitem caracterizar, classificar ou agrupar os indivíduos de uma mesma espécie em raças ou tipos distintos. Tais características podem ser definidas como uma particularidade individual, que em maior ou menor grau de variação, determina o tipo de raça ou tipo étnico a qual pertence (Rodero et al. 1992, pp.519-30; Bedotti et al. 2004, pp.261-71).

As medidas biométricas são tomadas com base no conhecimento que há grupos genéticos caprinos pernaltas e anões, caprinos de orelhas longas e caídas e outros de orelhas eretas e curtas. Além disso, o baixo custo na coleta e processamento dos dados biométricos, juntamente com o poder discriminante da análise multivariada, permitem preconizá-las como técnicas auxiliares no discernimento de populações caprinas. Estas medidas variam entre grupos genéticos e também se prestam à identificação racial, sendo utilizadas para classificação de caprinos desde a segunda metade do século passado. Para aprimorar estes estudos passou-se a realizar a mensuração simultânea de várias características e estabelecer índices entre medidas corporais (Bourzat et al. 1993, pp.667-74; Bouchel et al. 1997, pp.37-46). Apesar de que hoje a caracterização genética é a principal fonte de informação nas análises de distintividade (Alderson, 2018), a caracterização fenotípica é uma ferramenta acessível e fácil de ser realizada em programas de conservação e melhoramento e os criadores são promotores de diversidade genética, e devem, portanto, serem consultados nos programas de conservação e melhoramento da raça. Devido à falta de informações sobre os caprinos de Cabo Verde, objetivou-se caracterizar e discernir fenotipicamente as populações caprinas de Cabo Verde por meio de marcadores biométricos.

\section{MATERIAL E MÉTODOS}

Os dados foram provenientes de 533 caprinos acima de dois anos de idade de cinco das dez principais ilhas e em 18 diferentes localidades. Na ilha do Fogo foram coletadas amostras em oito diferentes localidades (Figura 1, Tabela I). Em cada rebanho se preencheu um formulário de pesquisa que reúne informações do fenótipo de cada animal amostrado, da propriedade e proprietário. Foram também feitas anotações quanto ao trânsito de animais, às raças exóticas utilizadas na caprinocultura e ao consumo de produtos de origem caprina.

A amostragem em Santiago foi reduzida em comparação às outras ilhas em estudo. Apesar do efetivo caprino ser maior na Ilha de Santiago (República de CaboVerde/MADRRM, 2004), seus rebanhos são menores. Em algumas das propriedades eles são compostos por um único animal. Nesta ilha, as unidades de produção possuem mais machos que fêmeas em idade reprodutiva, devido à tradição local de consumir carne de cabritos e bodes em datas festivas. Nas ilhas do Fogo e Santo Antão, há rebanhos mais numerosos que nas demais, o que possibilitou maior número de animais amostrados nestas ilhas.

Para obter as medidas lineares corporais foi utilizada uma fita métrica, enquanto os caprinos eram mantidos em posição correta de aprumos. As 16 características avaliadas foram: altura de cernelha (AC), distância da parte mais alta da cernelha ao chão; altura das patas (AP); altura da garupa ao chão (AG); comprimento corporal (CC), distância entre a parte cranial da tuberosidade maior do úmero e a tuberosidade isquiática; comprimento de orelha (CO); circunferência

Tabela I. Caprinos amostrados em cinco ilhas da República de Cabo Verde (Goats sampled on five islands in the Republic of Cape Verde).

\begin{tabular}{|c|c|c|c|}
\hline Ilha & $\mathrm{N}^{\circ}$ de animais & Concelho & Localidades \\
\hline \multirow{2}{*}{ Fogo } & 195 & São Filipe & $\begin{array}{c}\text { Vicente Dias, Genebra, Salto, Rocha Branca, Salto Acima, Monte } \\
\text { Grande }\end{array}$ \\
\hline & & $\begin{array}{l}\text { Santa Catarina } \\
\text { do Fogo }\end{array}$ & Achada Furna, Cabeça Fundão \\
\hline Santiago & 28 & $\begin{array}{c}\text { Tarrafal } \\
\text { São Miguel }\end{array}$ & $\begin{array}{c}\text { Tarrafal } \\
\text { Achada Pizarra }\end{array}$ \\
\hline São Nicolau & 64 & Ribeira Brava & Boqueirão, Caleijão \\
\hline São Vicente & 64 & São Vicente & $\begin{array}{l}\text { Ribeira do Calhau, Curral Tortolho, Ribeira de Vinha e Ribeira de } \\
\text { Julião }\end{array}$ \\
\hline Santo Antão & 182 & Porto Novo & Ponte Sul e Aureliano \\
\hline TOTAL & 533 & & 18 \\
\hline
\end{tabular}




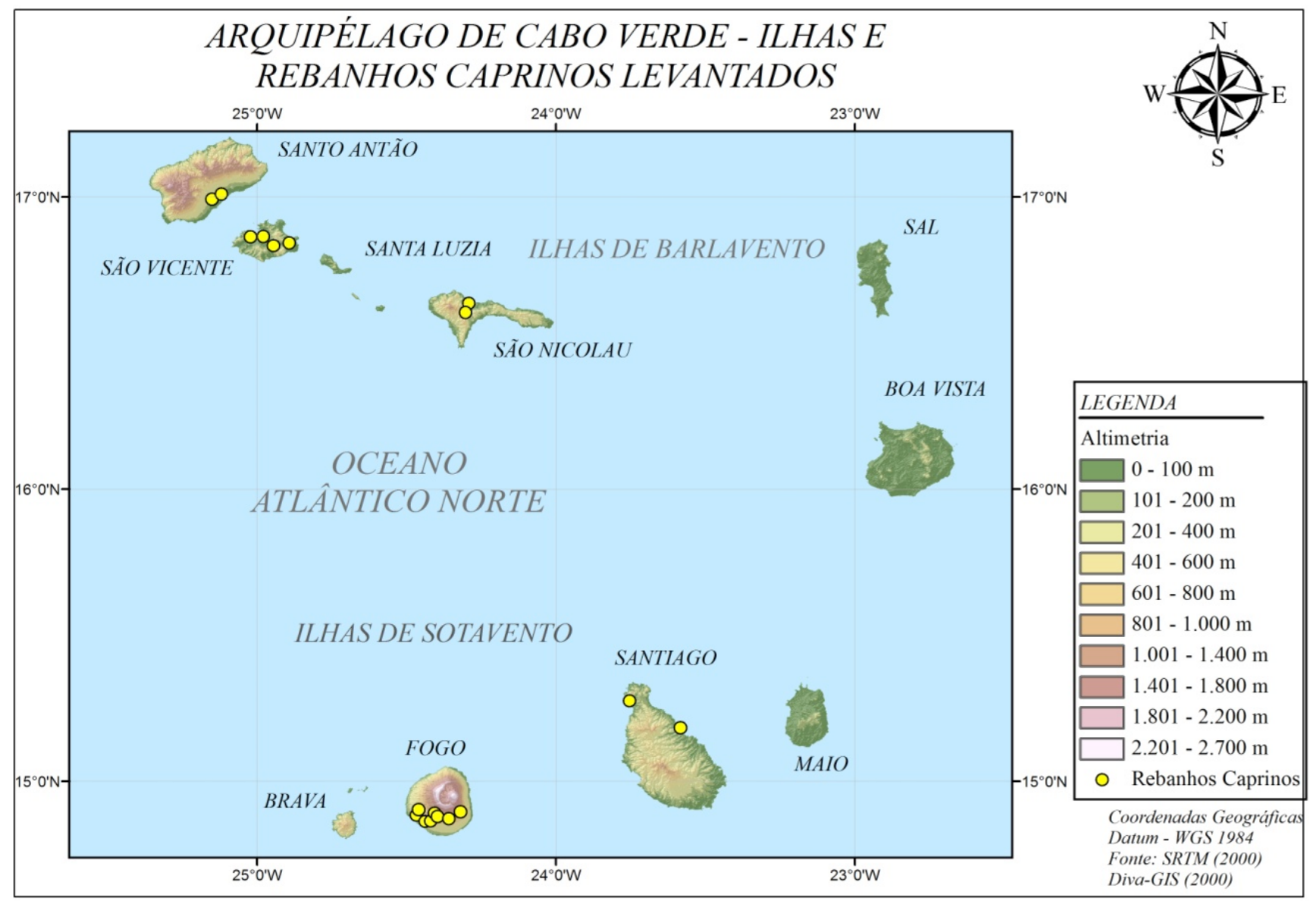

Figura 1. Localização das principais ilhas da República de Cabo Verde e distribuição geográfica das localidades dentro das ilhas de Cabo Verde onde foi realizada a amostragem (Location of the main islands of the Republic of Cape Verde and geographical distribution of the localities within the islands of Cape Verde where the sampling was carried out).

torácica $(\mathrm{CT})$, circunferência externa do tórax, ao nível da cernelha; comprimento da cabeça (CCAB), medida desde o occipital até o lábio inferior; longitude do rosto (LR), medida entre a linha imaginária que une o ângulo interno dos olhos e o ponto mais rostral da parte nasal; largura da cabeça (LCAB), medida entre os arcos zigomáticos; largura da garupa entre ílios (LIL), distância entre as protuberâncias ilíacas; largura da garupa entre ísquios (LIS), distância entre as pontas dos ísquios; comprimento da garupa (CGA), distância entre a tuberosidade coxal do ílio e a tuberosidade isquiática ou anca-jarrete; perímetro da canela (PCA), medida do terço médio da região metacarpiana do membro posterior esquerdo; tamanho do chifre (TCH); diâmetro do chifre $(\mathrm{DCH})$. A profundidade torácica (PT) foi calculada pela diferença entre duas medidas (AC - AP).

Os dados biométricos foram tabulados em planilhas do Excel e posteriormente analisados por da estatística descritiva, análise da variância, correlações entre as variáveis e o teste Student Newman Keuls para comparação das médias das diferentes populações (raças e tipos caprinos). Na análise de variância foi examinado o efeito da população sobre as medidas biométricas. Preliminarmente, foi realizado o teste do número de condições (NC), de acordo Montgomery, Peck \&Vining (2012, p.528), para diagnóstico do efeito da multicolinearidade. Após essa análise, foi identificada e descartada a variável PT, que provocou forte multicolinearidade nas medidas corpo- rais. As demais 15 variáveis foram padronizadas e submetidas às análises de agrupamento e de componentes principais. O critério para descarte de variáveis na análise de componentes principais (ACP) seguiu as recomendações de Jolliffe (1973, pp.21-31). Estabeleceu-se que o número de variáveis descartadas devia ser igual ao de componentes cuja variância (autovalor) é inferior a 0,7.

A análise de agrupamento foi conduzida adotando-se a distância generalizada de Mahalanobis $\left(\mathrm{D}^{2}\right)$ como medida de dissimilaridade, e foi empregado o método de agrupamento hierárquico Unweighted Pair Group Method With Arithmetic Mean (UPGMA) para gerar os agrupamentos e, consequentemente, o dendrograma. Para a formação de grupos mutuamente exclusivos empregou-se o método de otimização de Tocher. Avaliou-se, também, a importância relativa das características para a divergência segundo metodologia de Singh (1981, pp.237-45), o coeficiente de correlação cofenética (CCC) para avaliar a distorção provocada pelo agrupamento das populações e, realizou-se o bootstrap com 1000 repetições para avaliar a acurácia dos agrupamentos.

As análises foram realizadas pelos programas SAS System for Windows NT, versão 8.0, licenciado pela Universidade Federal de Viçosa (SAS, 1999) e GENES-versão 6.0 (Cruz, 2008).

\section{RESULTADOS E DISCUSSÃO}


Tabela II. Resumo da análise descritiva e de variância para medidas corporais $(\mathrm{cm})$ em cinco populações caprinas da República de Cabo Verde (Summary of the descriptive and variance analysis for body measurements (cm) in five goat populations of the Republic of Cape Verde).

\begin{tabular}{|c|c|c|c|c|c|c|c|c|c|c|c|c|c|c|c|c|}
\hline & $A C$ & $A P$ & PT & $\mathrm{CC}$ & $\mathrm{CO}$ & $A G$ & CT & CCAB & LR & LCAB & LIL & LIS & CGA & PCA & $\mathrm{TCH}$ & $\mathrm{DCH}$ \\
\hline Illhas & $* \star \star *$ & $* * *$ & $* \star *$ & $* \star \star *$ & $* \star *$ & * & $\star *$ & $* \star *$ & * & $\star \star * *$ & $\star * *$ & 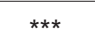 & $\star * * *$ & $* * *$ & $* \star *$ & ns \\
\hline CV & 5,90 & 9,61 & 10,31 & 7,50 & 9,44 & 6,09 & 7,11 & 6,26 & 8,11 & 7,36 & 8,94 & 9,64 & 7,25 & 8,22 & 22,85 & 12,28 \\
\hline Média & 66,16 & 35,60 & 30,55 & 65,18 & 13,92 & 68,73 & 78,84 & 19,82 & 15,62 & 14,06 & 15,39 & 16,42 & 14,83 & 8,57 & 20,29 & 9,01 \\
\hline $\begin{array}{l}\text { Desvio- } \\
\text { padrão }\end{array}$ & 3,90 & 3,42 & 3,15 & 4,89 & 1,31 & 4,18 & 5,60 & 1,24 & 1,27 & 1,04 & 1,38 & 1,58 & 1,07 & 0,70 & 4,64 & 1,11 \\
\hline
\end{tabular}

AC: altura de cernelha; AP: altura das patas; PT: profundidade torácica; CC: comprimento corporal; CO: comprimento da orelha; AG: altura da garupa; CT: circunferência torácica; CCAB: comprimento da cabeça; LR: longitude do rosto; LCAB: largura da cabeça; LIL: largura da garupa entre ílios; LIS: largura da garupa entre ísquios; CGA: comprimento de garupa; PCA: perímetro da canela; TCH: tamanho do chifre; DHC: diâmetro do chifre; CV: coeficiente de variação; * $P<0,05$; ** $P<0,01$; *** $P<0,001$; e ns: não-significativo.

Pelas anotações realizadas em campo, verificou-se que em praticamente todos os rebanhos avaliados, os reprodutores são oriundos do próprio rebanho, o que está de acordo com Ayantunde et al. (2007, p.143), analisando os sistemas de criação ovinos, caprinos e bovinos no Oeste da África, em que observaram que cerca de $75 \%$ dos animais advêm do próprio rebanho, prática que contribui para aumento de endogamia.

Na ilha do Fogo observou-se grande variabilidade dos caprinos quanto à sua forma e aptidão física, promovida pelo sistema de criação baseado no uso comum de recursos forrageiros locais, que facilita a conexão natural entre rebanhos próximos, proporcionando fluxo gênico. Verificou-se que nas localidades com maiores altitudes nesta ilha, os sistemas de criação apresentaram melhor aptidão dos rebanhos. Estes rebanhos caprinos são visados para produção leiteira e, no próprio local, os criadores produzem queijos de sabores peculiares, provavelmente devido às pastagens de campos altos com espécies nativas, ou mesmo endêmicas. O projeto de Proteção dos Recursos Naturais na Ilha do Fogo proporcionou este melhor desenvolvimento da caprinocultura na ilha, principalmente nas áreas de maior altitude, através da construção de currais melhorados, bebedouros, laticínios familiares, recuperação de pastagens degradadas e melhoria dos animais com introdução de bodes das ilhas Canárias.
Após estes investimentos, a pecuária na ilha do Fogo deixou de ser uma atividade tradicional, para ser uma atividade econômica sustentável (Inforpress, 2011).

Observou-se que os criadores das diferentes ilhas de Cabo Verde possuem preferência por reprodutores da raça Majorera oriundos das ilhas Canárias ou cruzamentos destes, devido, na opinião deles, terem as melhores características físicas para um bom reprodutor e por possuírem muitas características comuns entre os tipos locais.

$\mathrm{Na}$ análise descritiva e de variância realizada para as diferentes medidas corporais nas cabras cabo-verdianas (Tabela II), observou-se que os coeficientes de variação $(\mathrm{CV})$, para todas as variáveis, variaram de 5,90 a $22,85 \%$. As medidas que apresentaram os maiores $\mathrm{CV}$ foram: tamanho do chifre $(22,85 \%)$ e diâmetro do chifre $(12,28 \%)$. Os CV, para a maioria das medidas corporais foram próximos a 10\%. Estes CV indicam grau de variação fenotípica entre os indivíduos coletados e a existência de variabilidade genética para as características estudadas, passíveis de melhoria a partir de programas de melhoramento genético intra-populacional.

O resultado da análise de variância das diferentes medidas corporais (Tabela II) mostrou diferença significativa entre as populações das cinco ilhas, para a maioria das medidas avaliadas, exceto para diâmetro

Tabela III. Médias estimadas pelos mínimos quadrados (lsmeans) para medidas corporais em cinco populações caprinas da República de Cabo Verde (Medium estimated by least squares (Ismeans) for body measurements in five goat populations of the Republic of Cape Verde).

\begin{tabular}{|c|c|c|c|c|c|c|c|c|c|c|c|c|c|c|c|c|}
\hline Ilha & $A C$ & AP & PT & $\mathrm{CC}$ & $\mathrm{CO}$ & $A G$ & CT & CCAB & LR & LCAB & LIL & LIS & CGA & PCA & $\mathrm{TCH}$ & $\mathrm{DCH}$ \\
\hline Fogo & $64,77^{\mathrm{b}}$ & $37,23^{a}$ & $27,53^{c}$ & $68,27^{a}$ & $14,62^{\mathrm{a}}$ & $68,37^{a b}$ & $78,30^{\mathrm{ab}}$ & $19,03^{c}$ & $15,68^{a b}$ & $14,50^{\mathrm{a}}$ & $16,04^{a}$ & $15,95^{\circ}$ & $15,67^{a}$ & $8,37^{b}$ & $20,79^{a b}$ & $9,05^{a}$ \\
\hline \multicolumn{17}{|l|}{ São } \\
\hline Nicolau & $67,79^{a}$ & $35,21^{\mathrm{b}}$ & $32,59^{a}$ & $61,09^{c}$ & $12,89^{b}$ & $68,82^{\mathrm{ab}}$ & $80,67^{a}$ & $19,86^{b}$ & $16,01^{a}$ & $14,66^{a}$ & $16,07^{a}$ & $19,05^{\mathrm{a}}$ & $14,41^{\mathrm{b}}$ & $9,14^{a}$ & $22,16^{a}$ & $9,11^{a}$ \\
\hline Santiago & $61,90^{c}$ & $32,08^{c}$ & $29,83^{b}$ & $61,55^{c}$ & $12,90^{b}$ & $67,20^{b}$ & $75,65^{b}$ & $18,98^{c}$ & $15,33^{b}$ & $13,33^{b}$ & $15,38^{\mathrm{ab}}$ & $17,53^{b}$ & $14,70^{b}$ & $8,68^{b}$ & $21,89^{a}$ & $9,08^{a}$ \\
\hline \multicolumn{17}{|l|}{ Santo } \\
\hline Antão & $66,76^{\mathrm{ab}}$ & $34,55^{b}$ & $32,21^{a}$ & $64,58^{b}$ & $13,51^{b}$ & $68,72^{\mathrm{ab}}$ & $78,76^{a}$ & $20,25^{\mathrm{ab}}$ & $15,62^{\mathrm{ab}}$ & $13,68^{b}$ & $14,99^{b}$ & $15,97^{c}$ & $14,28^{b}$ & $8,61^{\mathrm{b}}$ & $18,82^{b}$ & $8,83^{a}$ \\
\hline \multicolumn{17}{|l|}{ São } \\
\hline Vicente & $67,95^{\mathrm{a}}$ & $35,82^{\mathrm{ab}}$ & $32,13^{a}$ & $63,65^{b c}$ & $14,55^{a}$ & $70,13^{a}$ & $79,83^{a}$ & $20,86^{a}$ & $15,21^{b}$ & $13,66^{b}$ & $14,21^{\mathrm{c}}$ & $16,16^{c}$ & $14,60^{\mathrm{b}}$ & $8,41^{b}$ & $20,57^{\mathrm{ab}}$ & $9,24^{a}$ \\
\hline
\end{tabular}

Teste de Student Newman Keuls para as medidas corporais. Letras diferentes na mesma coluna indicam diferença significativa entre as medidas. AC: altura de cernelha; AP: altura das patas; PT: profundidade torácica; CC: comprimento corporal; CO: comprimento da orelha; AG: altura da garupa; CT: circunferência torácica; CCAB: comprimento da cabeça; LR: longitude do rosto; LCAB: largura da cabeça; LIL: largura da garupa entre ílios; LIS: largura da garupa entre ísquios; CGA: comprimento de garupa; PCA: perímetro da canela; TCH: tamanho do chifre; e DHC: diâmetro do chifre. 


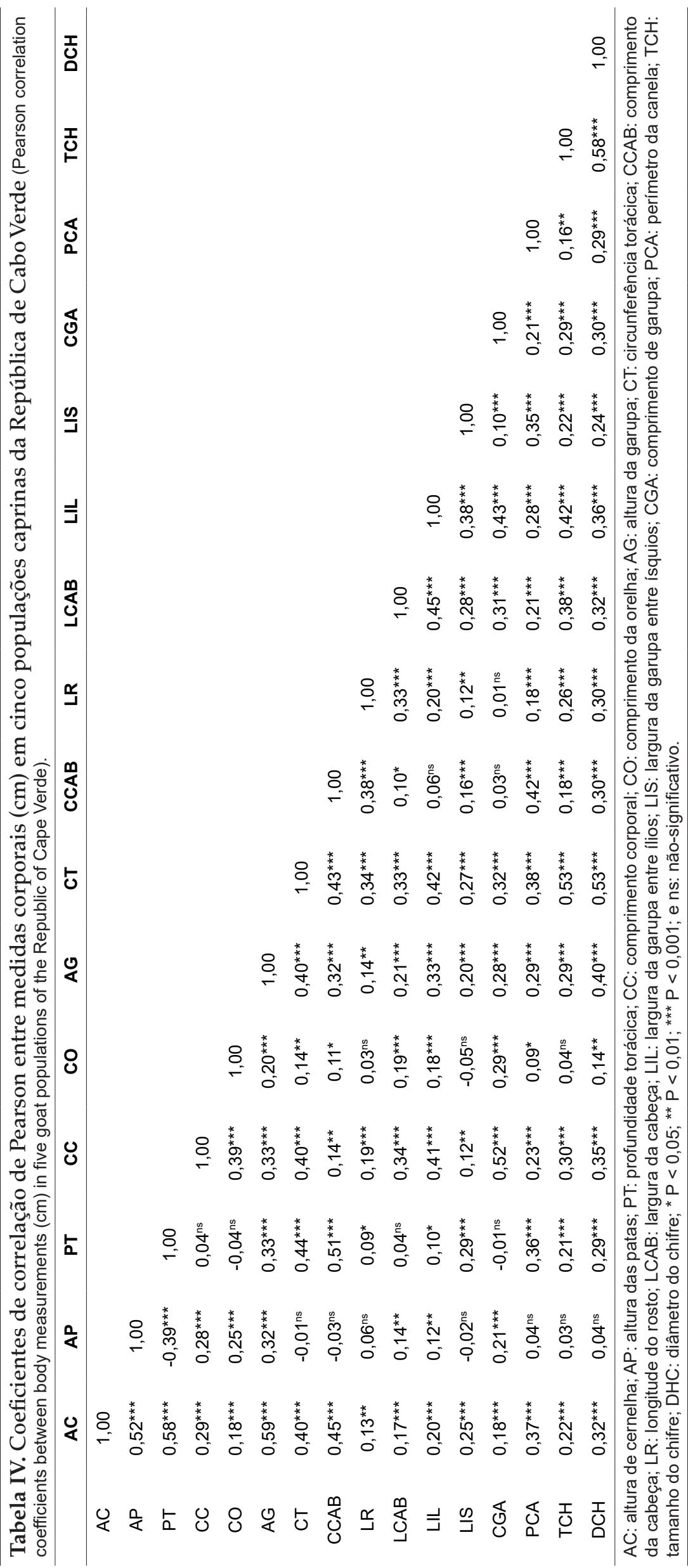

do chifre (DCH). De acordo com as médias das características das diferentes populações caprinas comparadas pelo teste de Student Newman Keuls (Tabela III), observou-se a formação de até três grupos de médias. As cabras da ilha do Fogo obtiveram as maiores médias para AP, CC, CO, e CGA e as menores médias para PT, LIS e PCA. Assim, elas se caracterizam por serem compridas $(\mathrm{CC}=68,27$ $\mathrm{cm})$, pouco profundas $(\mathrm{PT}=27,53$ $\mathrm{cm})$, pernaltas $(\mathrm{AP}=37,23 \mathrm{~cm})$, de altura mediana $(\mathrm{AC}=64,77 \mathrm{~cm})$, de orelhas de comprimento mediano $(\mathrm{CO}=14,62 \mathrm{~cm})$, linha dorsal não retilínea, ou seja, possui a garupa mais alta que a cernelha. Poder-se ia supor a possibilidade de uma maior deposição de carne na garupa devido ao maior comprimento da garupa $(C G A=15,67 \mathrm{~cm})$ comparado aos caprinos das demais ilhas.

As cabras da ilha de São Nicolau apresentam as maiores médias para as variáveis $\mathrm{PT}, \mathrm{CT}, \mathrm{LR}, \mathrm{LCAB}$, LIL, LIS, PCA e TCH, e as menores médias para CC e CO. Assim, elas se caracterizam por serem as mais profundas $(\mathrm{PT}=32,59 \mathrm{~cm})$, com a maior circunferência torácica (CT $=80,67 \mathrm{~cm})$ e com o maior tamanho dos chifres $(\mathrm{TCH}=22,16 \mathrm{~cm}$ ) dentre as ilhas em estudo. Como a CT está correlacionada com maior peso corporal, pode-se concluir que as cabras de São Nicolau possuem maior peso à idade adulta em comparação as demais.

$\mathrm{Na}$ ilha de Santiago, as cabras são as mais baixas $(\mathrm{AC}=61,90 \mathrm{~cm})$ e possuem as menores: altura das patas $(\mathrm{AP}=32,08 \mathrm{~cm})$; altura da garupa $(A G=67,20 \mathrm{~cm})$; circunferência torácica $(\mathrm{CT}=75,65 \mathrm{~cm})$; comprimento da cabeça (CCAB $=18,98 \mathrm{~cm}$ ); e largura da cabeça $(\mathrm{LCAB}=13,33 \mathrm{~cm})$ em relação às demais ilhas. As cabras da ilha de Santo Antão possuem as menores médias para as características comprimento da garupa (CGA = $14,28 \mathrm{~cm})$, tamanho do chifre (TCH $=18,82 \mathrm{~cm}$ ) e diâmetro do chifre $(\mathrm{DCH}=8,83 \mathrm{~cm}$ ). Para AC e PT estas cabras obtiveram valores próximos aos encontrados por Bouchel et al. (1997, pp.37-46) em caprinos do Rove, França, com média para AC de 66,5 cm e para PT de 32,3 $\mathrm{cm}$. Na ilha de São Vicente, as ca- 
bras possuem maior altura da garupa $(A G=70,13 \mathrm{~cm})$, comprimento da cabeça $(\mathrm{CCAB}=20,86 \mathrm{~cm})$, diâmetro do chifre $(\mathrm{DCH}=9,24 \mathrm{~cm})$ e altura de cernelha $(\mathrm{AC}=$ $67,95 \mathrm{~cm}$ ), caracterizando como as mais altas. Possuem também as menores médias para longitude do rosto $(\mathrm{LR}=15,21 \mathrm{~cm})$ e largura de garupa entre ílios $(\mathrm{LIL}=$ $14,21 \mathrm{~cm})$.

As cabras de Cabo Verde avaliadas, em relação aos caprinos das ilhas Canárias estudados por Capote et al. (1998, pp.168-69), obtiveram as menores médias para as características: comprimento corporal (CC), altura de garupa $(A G)$ e circunferência torácica $(C T)$. Para altura das patas (AP), altura de cernelha (AC), largura (LCAB) e comprimento da cabeça (CCAB), as cabras de ambos arquipélagos foram similares entre si. $\mathrm{O} \mathrm{TCH}$ das cabras cabo-verdianas variaram de 18,82 a 22,15 cm e foi, aproximadamente, similar ao encontrado por Machado et al. (1998, p.363) nos caprinos norte-africanos $(20,5 \pm 5,30 \mathrm{~cm})$. As cabras de Cabo Verde são pequenas se comparadas com os valores de 68 a $78 \mathrm{~cm}$ de altura de cernelha das raças espanholas (Herrera et al. 1996, p.41; Capote et al. 1998, p.168).Contudo, os valores da AC foram similares aos das cabras do Rove e das mexicanas (Bouchel et al. 1997, p.40; Lauvergne et al. 1997, p.503; Vargas et al. 2005, p.531). Em comparação com os caprinos do Norte de Camarões avaliados por Bourzat et al. (1993, p. 671), as cabras cabo-verdianas apresentaram maior estatura e foram mais profundas, pois o AC e PT os caprinos africanos foram, respectivamente, $50,2 \mathrm{~cm}$ e $23,5 \mathrm{~cm}$.

As correlações de Pearson entre as características avaliadas foram em grande parte significativas $(\mathrm{P}<0,05)$, positivas e de baixa à média magnitude (Tabela IV). A maioria das correlações negativas ob- servadas foram não-significativas, a única negativa e significativa foi entre AP e PT $(-0,39)$, uma vez que AC é a soma AP e PT. As maiores correlações foram entre: AC e AG $(0,59)$; AC e PT $(0,58)$; e TCH e DCH $(0,58)$. Já as menores correlações significativas foram entre: CO e PCA $(0,09)$; e PT e LR $(0,09)$. As correlações observadas foram, geralmente, inferiores as encontradas por Teixeira et al. (2000, p. 184) e Ribeiro et al. (2004, p. 339). A correlação entre AC e CC observado nas cabras cabo-verdianas foi similar ao encontrado por Ribeiro et al. 2004, p. 339 para Graúna $(0,28)$ e Moxotó $(0,21)$, e foi inferior ao observado para raça Canindé $(0,47)$.

As características intrínsecas a cada população, ou seja, as características mais relacionadas com as populações, foram CCAB, LR, LCAB, CO e PC. Isso porque elas possuem de baixa à média correlação com outras medidas, já que são mais influenciadas pela população, do que pela criação ou ambiente, fato também observado por Carneiro et al. (2010, pp. 58-65) em ovinos e por Silva (2007, pp.65-75) em caprinos, onde as medidas corporais AC, PT, AG e CT apresentaram as maiores correlações entre si, pois sofrem maior influência do ambiente, regime alimentar e sistema de criação.

Médias e correlações observadas entre as variáveis (Tabelas II, III e IV) justificam o uso de componentes principais para averiguar o grau de importância das características para a variabilidade total nos dados e para discriminação dos indivíduos no diagrama de dispersão.

De acordo com os resultados obtidos para os componentes principais, seus respectivos autovalores e porcentagens da variância explicada (Tabela V), observa-se que dos 15 componentes principais, nove $(60 \%)$ apresentaram variância (autovalor) inferior a 0,7 . Por-

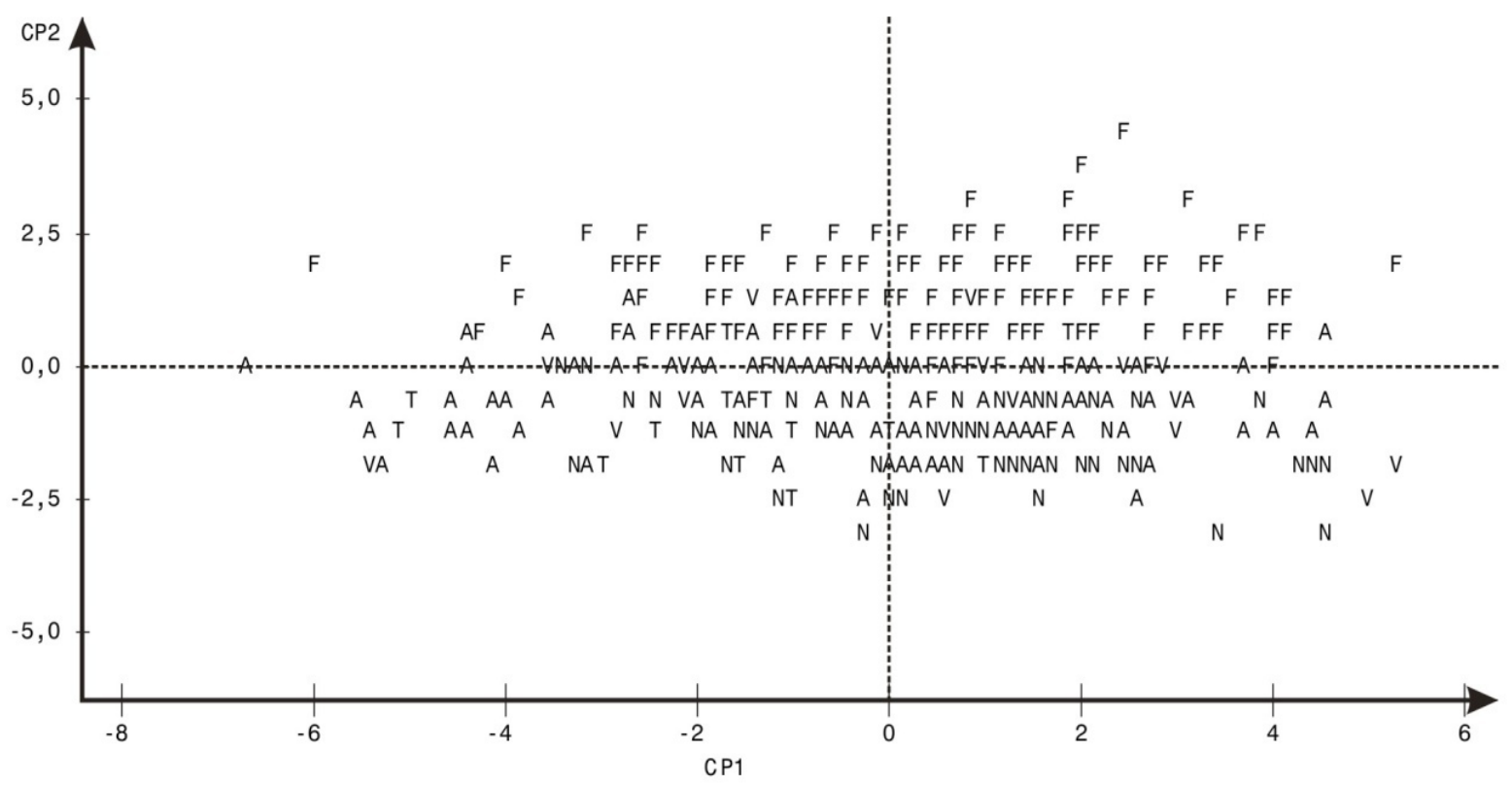

Figura 2. Dispersão gráfica dos escores das cinco populações de cabras na República de Cabo Verde, em relação aos componentes principais (CP) 1 (abscissa) e 2 (coordenada). Populaçôes: F: Ilha do Fogo; N: Ilha de São Nicolau; T: Santiago; A: Santo Antão; V São Vicente (Graphical dispersion of the five goat populations in the Republic of Cape Verde, in relation to the main components (CP) 1 (abscissa) and 2 (coordinate). Populations: F: Ilha do Fogo; N: St. Nicholas Island; T: Santiago; A: Santo Antão; V São Vicente). 
Tabela V. Componentes Principais (CP), autovalores $(\lambda i)$ e porcentagem da variância explicada pelos componentes das características mensuradas em caprinos da República de Cabo Verde (Main Components (CP) eigenvalues $\left(\lambda_{i}\right)$ and percentage of variance explained by the components of the characteristics measured in goats of the Republic of Cape Verde).

\begin{tabular}{lccc}
\hline $\mathrm{CP}$ & $\lambda_{\mathrm{i}}$ & $\begin{array}{c}\text { Variação } \\
\text { simples }(\%)\end{array}$ & $\begin{array}{c}\text { Variação } \\
\text { acumulada }(\%)\end{array}$ \\
\hline $\mathrm{CP}_{1}$ & 4,64 & 30,96 & 30,96 \\
$\mathrm{CP}_{2}$ & 1,71 & 11,43 & 42,39 \\
$\mathrm{CP}_{3}$ & 1,58 & 10,56 & 52,95 \\
$\mathrm{CP}_{4}$ & 1,17 & 7,81 & 60,75 \\
$\mathrm{CP}_{5}$ & 0,96 & 6,42 & 67,17 \\
$\mathrm{CP}_{6}$ & 0,90 & 5,98 & 73,15 \\
$\mathrm{CP}_{7}$ & 0,65 & 4,31 & 77,46 \\
$\mathrm{CP}_{8}$ & 0,53 & 3,54 & 81,00 \\
$\mathrm{CP}_{9}$ & 0,52 & 3,47 & 84,47 \\
$\mathrm{CP}_{10}$ & 0,49 & 3,27 & 87,74 \\
$\mathrm{CP}_{11}$ & 0,47 & 3,13 & 90,87 \\
$\mathrm{CP}_{12}$ & 0,41 & 2,76 & 93,63 \\
$\mathrm{CP}_{13}$ & 0,38 & 2,51 & 96,14 \\
$\mathrm{CP}_{14}$ & 0,35 & 2,33 & 98,48 \\
$\mathrm{CP}_{15}$ & 0,23 & 1,52 & 100,00 \\
\hline & & &
\end{tabular}

tanto, as nove variáveis que apresentarem maiores coeficientes, em valor absoluto, a partir do último componente principal, são passíveis de descarte, conforme apresentado na Tabela VI. Para os rebanhos estudados, os seis primeiros componentes principais foram se- lecionados, explicando $73,15 \%$ da variação total dos dados avaliados (Tabela V).

As características em ordem de menor importância para explicar a variação total, foram: AC, TCH, CT, CC, CGA, PCA, DCH, LIL e CO (Tabela VI). Considerando os resultados, verifica-se que as variáveis mais importantes para discriminar os indivíduos neste estudo foram: AP, AG, CCAB, LR, LCAB e LIS. Entre as 15 variáveis avaliadas, apenas seis contribuíram para uma maior discriminação das populações. Para outro conjunto de populações, diferente desta amostragem, os resultados das características mais ou menos discriminantes, podem diferir deste resultado, necessitando investigar cada situação.

Observa-se que as características de menor importância para explicar a variação total apresentaram correlações lineares simples e significativas com as demais (Tabelas IV e VI). Manly (2008, pp.89-104) ressalta que, quanto menor a correlação existente entre as variáveis, mais componentes serão necessários para explicar a variação total dos dados e, se tais correlações forem muito baixas, esse tipo de estudo não faz sentido. Portanto, a menor variação acumulada nos componentes principais, no presente estudo, se deve as baixas e médias correlações entre as variáveis.

No diagrama de dispersão gráfica (Figura 2) observou-se que as cabras do Fogo encontraram-se predominantemente nos dois quadrantes superiores, especialmente direito, enquanto as cabras de São Nicolau encontram-se em ambos quadrantes inferiores e predominaram no inferior direito. As cabras de Santo Antão se posicionaram nos quadrantes inferiores. Os

Tabela VI. Coeficientes de ponderação das variáveis com os nove componentes principais (CP's) menos importantes para explicar a variação total das características mensuradas em caprinos de Cabo Verde (Weighting coefficients of the variables with the nine major components (CPs) less important to explain the total variation of the characteristics measured in Cape Verde goats).

\begin{tabular}{|c|c|c|c|c|c|c|c|c|c|}
\hline & $\mathrm{CP}_{7}$ & $\mathrm{CP}_{8}$ & $\mathrm{CP}_{9}$ & $\mathrm{CP}_{10}$ & $\mathrm{CP}_{11}$ & $\mathrm{CP}_{12}$ & $\mathrm{CP}_{13}$ & $\mathrm{CP}_{14}$ & $\mathrm{CP}_{15}$ \\
\hline$A C$ & 0,03 & $-0,18$ & $-0,07$ & 0,19 & $-0,04$ & 0,00 & 0,04 & $-0,16$ & $-0,71$ \\
\hline AP & $-0,14$ & $-0,14$ & 0,24 & 0,10 & $-0,20$ & 0,14 & 0,00 & $-0,03$ & 0,49 \\
\hline $\mathrm{CC}$ & $-0,36$ & $-0,08$ & 0,14 & 0,06 & $-0,05$ & $-0,71$ & $-0,10$ & 0,16 & $-0,06$ \\
\hline $\mathrm{CO}$ & 0,65 & 0,11 & 0,16 & 0,11 & $-0,04$ & 0,18 & $-0,11$ & 0,07 & $-0,05$ \\
\hline$A G$ & 0,11 & 0,47 & $-0,32$ & $-0,39$ & 0,30 & $-0,11$ & $-0,16$ & 0,27 & 0,15 \\
\hline CT & $-0,09$ & $-0,07$ & $-0,31$ & 0,25 & $-0,28$ & 0,16 & $-0,59$ & $-0,30$ & 0,22 \\
\hline CCAB & 0,01 & $-0,05$ & $-0,12$ & 0,31 & 0,23 & $-0,12$ & 0,50 & $-0,05$ & 0,35 \\
\hline LR & $-0,24$ & 0,39 & 0,23 & 0,01 & 0,08 & 0,16 & $-0,18$ & 0,10 & $-0,20$ \\
\hline LCAB & 0,26 & $-0,40$ & $-0,44$ & $-0,16$ & 0,23 & $-0,16$ & $-0,01$ & $-0,10$ & 0,02 \\
\hline LIL & 0,02 & 0,51 & $-0,17$ & 0,07 & $-0,47$ & $-0,04$ & 0,43 & $-0,21$ & $-0,03$ \\
\hline LIS & 0,17 & 0,11 & 0,36 & 0,35 & 0,29 & $-0,13$ & $-0,26$ & 0,06 & 0,08 \\
\hline CGA & $-0,43$ & $-0,04$ & $-0,05$ & 0,05 & 0,48 & 0,51 & 0,15 & $-0,06$ & $-0,07$ \\
\hline PCA & $-0,12$ & $-0,25$ & 0,10 & $-0,51$ & $-0,33$ & 0,20 & 0,00 & 0,26 & 0,01 \\
\hline $\mathrm{TCH}$ & 0,15 & $-0,23$ & 0,08 & 0,21 & $-0,13$ & 0,15 & 0,18 & 0,63 & $-0,03$ \\
\hline $\mathrm{DCH}$ & 0,17 & $-0,09$ & 0,51 & $-0,40$ & 0,12 & $-0,07$ & 0,11 & $-0,49$ & 0,04 \\
\hline
\end{tabular}

AC: altura de cernelha; AP: altura das patas; CC: comprimento corporal; CO: comprimento da orelha; AG: altura da garupa; CT: circunferência torácica; CCAB: comprimento da cabeça; LR: Iongitude do rosto; LCAB: largura da cabeça; LIL: largura da garupa entre ílios; LIS: largura da garupa entre ísquios; CGA: comprimento de garupa; PCA: perímetro da canela; TCH: tamanho do chifre; e DHC: diâmetro do chifre. 


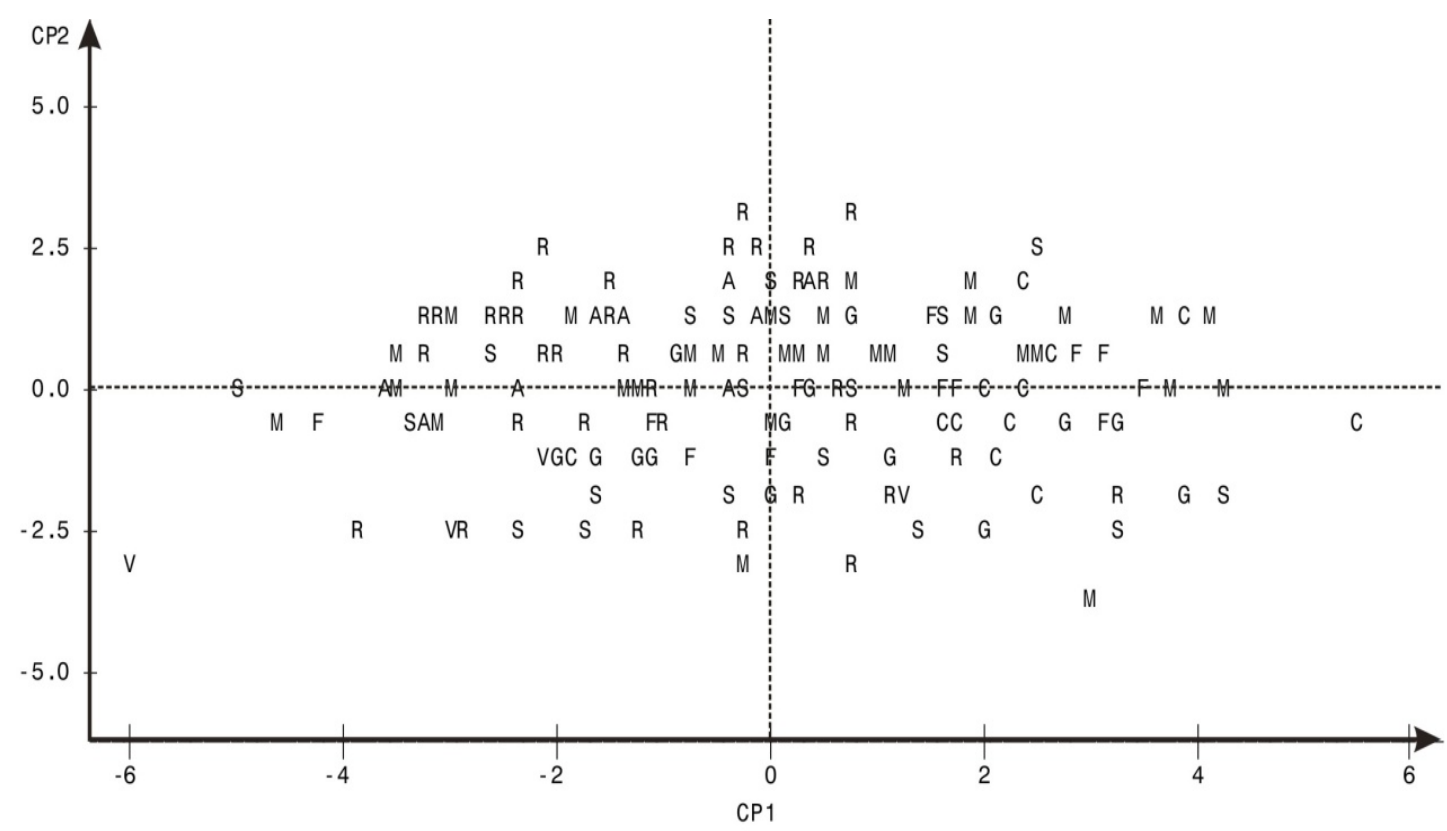

Figura 3. Dispersão gráfica dos escores da população de cabras de oito localidades da ilha do Fogo, em rela-

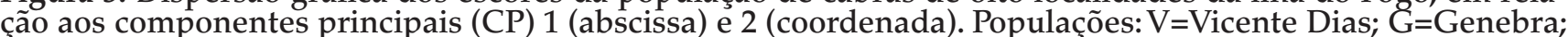
S=Salto; R=Rocha Branca; A=Salto Acima; M=Monte Grande; F=Achada Furna; C=Cabeça Fundão (Graphic dispersion of goat population scores from eight localities of Fogo Island, relative to the main components (CP) 1 (abscissa) and 2 (coordinate). Populations: V=Vicente Dias; G=Genebra; S=Salto; R=Rocha Branca; $A=$ Salto Acima; M=Monte Grande; F=Achada Furna; C=Cabeça Fundão.)

caprinos de Santiago encontram-se no quadrante inferior esquerdo em maior proximidade com as cabras das ilhas de Barlavento (São Nicolau, Santo Antão e São Vicente). As cabras de Santiago foram biometricamente mais similares as cabras das ilhas do norte, ou Barlavento, que a vizinha ilha do Fogo, ou Sotavento. Isso deve ser explicado por outros fatores que possibilitaram o intercâmbio entre estas ilhas. Mesmo com maior proximidade geográfica entre Santiago e Fogo observou-se maior distanciamento entre estas cabras por meio deste estudo com dados biométricos e ACP. As cabras de São Vicente ocuparam os quadrantes inferiores, especialmente o direito. Caprinos oriundos das ilhas de Barlavento (Santo Antão, São Vicente e São Nicolau) agruparam-se na Figura 2 à semelhança da proximidade física das ilhas que povoam.

Este tipo de resultado serve como subsídio para uma futura homologação, perante o Ministério da Agricultura de Cabo Verde, e conservação dos grupos genéticos caprinos existentes nas ilhas. Isso é importante para o Cabo Verde, que ainda não possui nenhum estudo da variabilidade genética molecular.

Na ilha do Fogo foram amostradas localidades com características bem distintas segundo a altitude. Os rebanhos de áreas com maior altitude possuem, teoricamente, melhores condições para a prática da caprinocultura leiteira devido, provavelmente, ao maior percentual de sangue exótico observado neste grupo genético oriundo da introdução de bodes Majoreros das ilhas Canárias (Inforpress, 2011). Em algumas localidades destas ilhas, principalmente as com maior altitude, está tendo absorção dos genótipos locais pela raça Majorera devido ao plano governamental de me- lhoramento genético, o que gera risco de perda da diversidade e do patrimônio genético. Diante destes fatos e também do distanciamento destes caprinos com os das demais ilhas em estudo, analisou-se, em particular, caprinos da ilha do Fogo com o objetivo de saber se eles são melhor ou pior conservados.

De acordo com o diagrama de dispersão gráfica (Figura 3), observa-se que os rebanhos da ilha do Fogo agruparam-se de acordo com a proximidade geográfica entre eles. Estes rebanhos possuem vasta variabilidade, o que pode ser observado devido à dispersão deles em vários quadrantes. $\mathrm{O}$ rebanho mais padronizado foi da localidade com maior altitude (cerca de $1064 \mathrm{~m}$ ) chamada Cabeça Fundão. Este rebanho possui maior infusão de sangue Majorera tendo fêmeas F1 e F2 com Majorera. Em proximidade a estes animais, encontram-se as cabras oriundas de outras regiões com elevada altitude também, como Achada Furna. Este maior percentual de sangue Majorera nestas localidades se deve ao projeto de raças melhoradas que levou a pecuária praticada no Fogo a outros patamares, principalmente para os criadores de caprinos de Cabeça do Monte, Ribeira Filipe, Cabeça Fundão, Chã das Caldeiras e outras localidades das zonas altas do Fogo, que já exportam raça caprina melhorada para as ilhas de Santiago, Boavista e Brava (A semana, 2011).

As cabras cabo-verdianas de $\mathrm{M}$ predominaram nos quadrantes superiores e as de $\mathrm{S}$ encontraram-se, praticamente, em mesmo número em cada quadrante (superior e inferior), ou seja, foram as mais dispersas; $\mathrm{R}$ encontraram-se em todos os quadrantes, mas predominaram no superior esquerdo; A é nitidamente majoritária no quadrante superior esquerdo; F está au- 
sente no quadrante superior esquerdo e predomina no superior direito; $C$ no quadrante direito inferior; $V$ nos quadrantes inferiores; $\mathrm{G}$ particularmente no inferior direito (Figura 3). Duas (M e F) das três subpopulações da Ilha do Fogo onde foram realizados cruzamentos com a raça Majorera, ficaram predominantemente no quadrante superior direito, e C no inferior direito. A e R predominaram no quadrante superior esquerdo e não foram cruzadas com a raça Majorera.

As medidas corporais consideradas nas análises de agrupamento foram $\mathrm{AC}, \mathrm{AP}, \mathrm{CC}, \mathrm{CO}, \mathrm{AG}, \mathrm{CT}, \mathrm{CCAB}$, LR, LCAB, LIL, LIS, CGA, PCA, TCH e DHC que apresentaram fraca multicolinearidade $(\mathrm{NC}<100)$ na amostra. Resultado semelhante foi encontrado em rebanhos espanhóis de caprinos nativos (Herrera et al. 1996, p. 46) em que a profundidade torácica não foi discriminante, não devendo, portanto, ser considerada na elaboração de estudos de diferenças raciais, e também por Pires et al. (2013), com caprinos brasileiros e marroquinos.

A análise de agrupamento, a partir das 15 medidas corporais das fêmeas adultas (acima de dois anos de idade), foi conduzida adotando-se a distância de Mahalanobis $\left(\mathrm{D}^{2}\right)$ como medida de dissimilaridade e o método UPGMA. Verificou-se na matriz da distância generalizada de Mahalanobis $\left(\mathrm{D}^{2}\right)$ que o valor máximo $D^{2}$ foi entre as populações das ilhas do Fogo e São Nicolau (22,73), as mais divergentes, enquanto o valor mínimo foi entre Santo Antão e São Vicente $(3,71)$, as mais similares (Tabela VII). Em ambos os casos a distância geográfica entre as ilhas pode explicar os resultados encontrados. Em outros contextos, como dos caprinos mexicanos, a análise discriminante com variáveis canônicas canônica tem oferecido também excelentes resultados nos agrupamentos (Sánchez Gutiérrez et al., 2018)

Com a utilização do método de agrupamento UPGMA, obteve-se CCC de 0,79 (Figura 4). A partir desse coeficiente, infere-se que a distância $\mathrm{D}^{2}$ foi adequada para resumir a informação do conjunto de dados e que o método UPGMA proporcionou uma fidedigna representação gráfica dos agrupamentos. A acurácia obtida

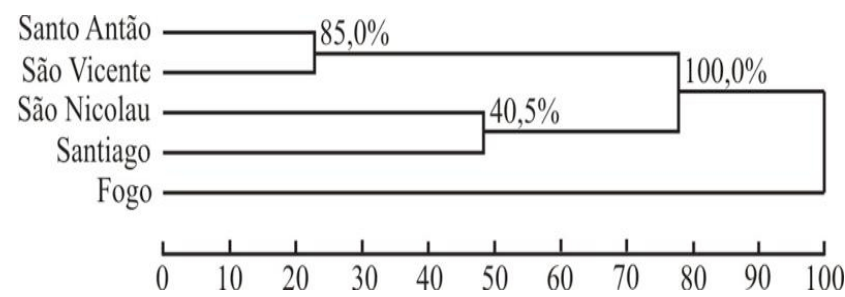

Figura 4. Dendrograma obtido a partir da distância generalizada de Mahalanobis e do algoritmo UPGMA, com base em caracteres biométricos evidenciando as relações entre cinco populações caprinas da República de Cabo Verde. As distâncias estão expressas em porcentagens na linha abaixo ao dendrograma e os valores de bootstrap são indicados em cada nó (Dendrogram obtained from the generalized distance of Mahalanobis and the algorithm UPGMA, based on biometric characters evidencing the relations among five caprine populations of the Republic of Cape Verde. The distances are expressed in percentages in the line below the dendrogram and the bootstrap values are indicated on each node). nas junções do dendrograma foi igual e superior a $85 \%$ em dois dos três nós formados.

O dendrograma a partir de 15 variáveis em cinco populações (Figura 4) colocou as cabras da ilha de Fogo em ramo a parte das demais com 100\% de acurácia. Agruparam-se num ramo as cabras das ilhas de Santo Antão e São Vicente com 85\% de acurácia, enquanto as de São Nicolau e Santiago apresentaram agrupamento incerto entre si, mas distintas das demais. Os agrupamentos das populações nas ilhas de Santo Antão e São Vicente denota que a proximidade geográfica de ambas foi importante para seu agrupamento, com suposta relação de parentesco entre estas populações devido ao trânsito de animais entre estas ilhas.

As cabras da ilha do Fogo são as mais distintas das demais, isto devido a sua distância geográfica. Elas apresentam melhor conformação corporal por estarem mais direcionados para a produção leiteira e são submetidas a um melhor manejo nutricional que as demais. Além disso, houve recentemente, a introdução de bodes das ilhas Canárias em certas localidades da ilha do Fogo.

No dendrograma (Figura 5) para as diferentes localidades da ilha do Fogo, observa-se que o agrupamento da localidade de Vicente Dias foi o mais divergente de todos. As localidades Monte Grande, Achada Furna e Cabeça Fundão agruparam-se formando um segundo ramo. Este agrupamento foi concordante com o gráfico de dispersão gráfica utilizando a ACP (Figura 3). A localidade Genebra formou um terceiro ramo. Salto Acima formou um agrupamento incerto com as localidades Salto e Rocha Branca.

Os resultados dos dendrogramas foram facilmente interpretáveis, mas não foi possível saber ao certo o número ótimo de grupos. Para contornar esta deficiência utilizou-se o método de otimização de Tocher que possibilitou verificar a formação de dois grupos distintos utilizando as $\mathrm{D}^{2}$. Um composto pelas populações das ilhas de Santo Antão, São Vicente, Santiago e São Nicolau; outro grupo composto somente pela população do Fogo. A formação dos grupos está de acordo com o dendrograma obtido (Figura 4). Verificou-se, portanto, que o método de otimização de Tocher e o método hierárquico (UPGMA) foram concordantes na partição dos grupos.

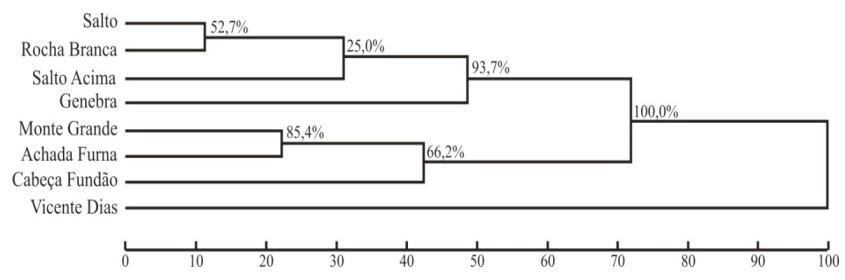

Figura 5. Dendrograma obtido a partir da distância generalizada de Mahalanobis e do algoritmo UPGMA, com base em caracteres biométricos evidenciando as relações entre caprinos de diferentes localidades da ilha do Fogo (Dendrogram obtained from the generalized distance of Mahalanobis and the algorithm UPGMA, based on biometric characters evidencing the relations among localities of Fogo Island). 
Todos os caracteres biométricos mensurados (AC, $\mathrm{AP}, \mathrm{CC}, \mathrm{CO}, \mathrm{AG}, \mathrm{CT}, \mathrm{CCAB}, \mathrm{LR}, \mathrm{LCAB}$, LIL, LIS, CGA, PCA, TCH e DHC) contribuíram para a determinação da divergência genética entre as populações, em maior ou menor proporção. Quanto à importância relativa das características para a divergência segundo a metodologia de Singh (1981), a medida da garupa entre ísquios apresentou maior contribuição relativa para a divergência $(18,66 \%)$, seguida das características comprimento da cabeça $(12,90 \%)$, comprimento corporal $(10,42 \%)$ e altura de cernelha $(9,73 \%)$.

\section{CONCLUSÃO}

Existem diferentes tipos caprinos cabo-verdianos. As populações caprinas das ilhas do Fogo, Santiago, São Nicolau e São Vicente apresentam características biométricas diferenciadas entre si. As cabras do Fogo são um grupo a parte das demais cabras cabo-verdianas. Era esperado que os grupos genéticos oriundos das ilhas de Sotavento se agrupassem, porém as cabras de Santiago são mais próximas das populações caprinas das ilhas de Barlavento. As populações caprinas das ilhas de Barlavento agrupam-se pelas distâncias geográficas. Os rebanhos da ilha do Fogo agrupam-se pela proximidade geográfica entre as localidades da ilha e dos locais onde são realizados cruzamentos com a raça Majorera de Canárias. Sugere-se aumentar o número de caprinos amostrados na ilha de Santiago e realizar estudos de caracterização genética por meio de marcadores moleculares para verificar, com maior acurácia, se a ocorrência destes agrupamentos com o uso de marcadores biométricos procede.

\section{AGRADECIMIENTOS}

Agradecimentos a CAPES, CNPq (Projeto 490488/2008-0), Empresa Brasileira de Pesquisa Agropecuária (EMBRAPA-CNPC) e a Direção Geral da Agricultura Silvicultura e Pecuária (DGASP-Cabo Verde) pelos auxílios concedidos.

\section{BIBLIOGRAFIA}

Alderson, GLH 2008, 'Conservation of breeds and maintenance of biodiversity: justification and methodology for the conservation of Animal Genetic Resources', Archivos de Zootecnia, vol. 67, no. 258, pp. 300-309.

ASemana 2011 , Ministério da Agricultura apoia criadores com reprodutores para melhorar raça de caprinos, Cape Verde, Jornal a Semana, viewed 28 July 2011, <http://www.asemana.publ.cr>

Ayantunde, AA, Kango, M, Hiernaux, P, Udo, HMJ \& Tabo, R 2007, 'Herders' Perceptions on Ruminant Livestock Breeds and Breeding Management in Southwestern Niger', Human Ecology, vol.35, no. 1, pp. 139-149.<https://doi.org/10.1007/s10745-006-9049-6>.

Bedotti, D, Gómez Castro, AG, Sánchez Rodriguez, M, \& Martos Peinado, J 2004, 'Caracterización morfológica y faneróptica de la cabra colorada pampeana', Archivos de Zootecnia, vol.53, no. 203-204, pp. 261-71.

Bouchel, D, Lauvergne, JJ, Guibert, E \& Minvielle, F 1997,'Étudemorpho-biométrique de la chèvre du rove. Hauteurau garrot (HT), profondeur du thorax (PT), videsous-sternal (VSS) et índice de gracilités ous-sternale (IGs) chezfemelles', Revue de Médecine Vétérinaire, vol. 148 , no. 1, pp 37-46.
Bourzat, D,Souvenir-Zafindrajaona, P,Lauvergne, JJ, et al. 1993, 'Comparaison morpho-biométrique dês chèvesau Nord Cameroun et au Tchad', Revued 'Élevage et de Médecine Vétérinaire des Pays Tropicaux, vol.46, no. 4, pp. 667-74.<http://revues.cirad.fr/index. $\mathrm{php/REMVT/issue/view/792>}$

Capote, J, Delado, JV, Fresno, M, Camacho, ME \& Molina, A 1998, 'Morphological variability in the Canary goat population', Small Ruminant Research, vol.27, pp.167-72.

Carneiro, H, Louvandini, H, Paiva, SR, Macedo, F, Mernies, B \& McManus, C 2010, 'Morphological characterization of sheep breeds in Brazil, Uruguay and Colombia', Small RuminantResearch, vol.94, pp.58-65.

Carvalho, IRTV \& Sá, AJ 2007, 'O retorno de emigrantes e o problema de reinserção em Cabo Verde', Revista de Geografia, vol.24, no.2, pp.121-35.

Cruz, CD Programa Genes: aplicativo computacional em genética. Viçosa: UFV, versão 6.0, 2008.

Faostat. Live animals. Disponível em: <http://faostat.fao.org.>. Acesso em: 15 dez. 2017

Herrera, M, Rodero, E, Gutierrez, MJ, Peña, F, Rodero, JM 1996, 'Application of multifactorial discriminant analysis in the morphostructural differentiation of Andalusian caprine breeds', Small Ruminant Research, vol. 22, no. 1, pp. 39-47.

Inforpress 2011, Agência de Notícias de Cabo Verde, viewed 30 July $2011,<$ http://www.inforpress.publ.cv>

Jolliffe, IT1973 'Discarding variables in a principal component analysis. II. Real data', Applied Statistics, vol.22, p.21-31

Lauvergne, JJ, Bouchel, D, Minvielle, F, Guibert, É 1997, 'Étude morpho-biométrique de la chèvre du Rove. II. Longueurd'oreille (LO) et indice auriculo-thoracique (IAt)', Revue de Médecine Vétérinaire, vol.148, no. 6, pp.501-510.

Machado, TMM, Lauvergne, JJ, Chakir, M, Souvenir-Zafindrajaona, P, Silva, HD 1998, 'Morfo-biometria no estudo comparativo de populações caprinas', Genetics and Molecular Biology, vol.21, no.3. (supplement), pp.363.

Machado, TMM $2013^{\prime}$ Os pequenos ruminantes na história da pecuária brasileira', Proceedings of the X Workshop de Produção de Caprinos na Região da Mata Atlântica, Embrapa, Juiz de Fora, pp. 11-45.

Manly, BJF 2008, Métodos estatísticos multivariados: Uma introdução, 3rd edn, Bookman, Porto Alegre.

Montgomery, DC, Peck, EA \& Vining, GG 2012, Introduction to Linear Regression Analysis, 5nd edn, John Wiley \& Sons, Inc.

Pecuária 2011, Criação de cabras de raça melhorada para produção de leite e derivados. Disponível em: <http://ie.ic.cv>. Acesso em:15 Julho 2011.

Silva, NV, Fraga, AB, Filho, JTA., Neto, CCC, Silva, FL., Costa, PPS \& Lira Junior, WB 2007, 'Caracterização Morfométrica de ovinos deslanados Cabugi e Morada Nova', Revista Científica de Produção Animal, vol. 9, n o. 65-75. 15July 2011, <http://ie.ic.cv>.

Pires, LC, Machado, TMM, Araújo, AM, Olson, TA, Silva, JBL, Torres, RA \& Costa, MS2012,'Biometricvariability of goat populations revealed by means of principal component analysis', Genetics and Molecular Biology, vol. 35, no.4, pp.777-782.

Pires, LC, Machado, TMM, Araújo, AM, Silva, JBL, Euclydes, RF, Costa, MS \&Olson, TA 2013,'Clusterevaluation of Brazilian and Moroccan goat populations using physical measurements', Revista Brasileira de Zootecnia, vol.42, pp.713-720.

República de Cabo Verde/MADRRM - Ministério Do Ambiente, do Desenvolvimento Rural e dos Recursos Marinhos. Recenseamento Geral 2004, Viewed 29 August 2011, <http://www.governo.cv>.

Ribeiro, MN, Silva, JV, Pimenta Filho, EC \& Sereno, JRB2004, 'Estúdio de las correlaciones entre características fenotípicas de caprinos naturalizados', Archivos de Zootecnia, vol.53, pp.337-340.

Rodero, E, Herrera, M \& Gutiérrez, MJ 1992, 'Morphostructural evolution of the Blanca Serrana caprine breed based of their crossing for milking aptitude', Archivos de Zootecnia, vol.41 (extra), pp.519-530. SAS/STAT. User'sguide. Versão 8.0. Cary: SAS Institut Inc., 1999. 
Sánchez Gutiérrez, R.A. et al. 2018. 'Caracterización morfológica de un rebaño de conservación de cabras criollas en Zacatecas, México', Archivos de Zootecnia, vol. 67, no.257, pp.73-79.

Silva, NV, Fraga, A., Filho, JTA, Neto, CCC, Silva, FL, Costa, PPS \& Lira Junior, WB 2007,'Caracterização Morfométrica de ovinos deslanados Cabugi e Morada Nova', Revista Científica de Produção Animal, vol. 9, pp. 65-75.

Singh, D 1981, 'The relative importance of characters affecting genetic divergence', Indian Journal of Genetics, vol.41, no.2, pp. 237-245.
Teixeira, MPB, Barros, NN, Araújo, AM, Villarroel, AS 2000, 'Relação entre medidas corporais e peso vivo em caprinos das raças Saanen e Anglo-Nubiana', Revista Científica de Produção Animal, vol.2, no.2, pp.178-189.

Vargas, S, Sánchez, M, Rodríguez, JJ, et al. 2005, 'La cabra para carne y su respuesta a la mejora del manejo', Archivos de Zootecnia, vol.54, pp.529-534. 